\title{
Formation and evolution of the Galactic bulge: constraints from stellar abundances
}

\author{
S. K. Ballero ${ }^{1}$, F. Matteucci ${ }^{1,2}$, L. Origlia ${ }^{3}$, and R. M. Rich ${ }^{4}$ \\ 1 Dipartimento di Astronomia, Universitá di Trieste, via G. B.Tiepolo 11, 34131 Trieste, Italy \\ e-mail: ballero@oats.inaf.it \\ 2 INAF - Osservatorio Astronomico di Trieste, via G. B.Tiepolo 11, 34131 Trieste, Italy \\ 3 INAF - Osservatorio Astronomico di Bologna, via G. Ranzani 1, 40127 Bologna, Italy \\ 4 Department of Physics and Astronomy, UCLA, 430 Portola Plaza, Box 951547, Los Angeles, CA 90095-1547, USA
}

Received 18 October 2006 / Accepted 29 January 2007

ABSTRACT

\begin{abstract}
Aims. We compute the chemical evolution of the Galactic bulge in the context of an inside-out model for the formation of the Milky Way. The model contains updated stellar yields from massive stars. The main purpose of the paper is to compare the predictions of this model with new observations of chemical abundance ratios and metallicity distributions in order to put constraints on the formation and evolution of the bulge.

Methods. We computed the evolution of several $\alpha$-elements and Fe and performed several tests by varying different parameters such as star formation efficiency, slope of the initial mass function and infall timescale. We also tested the effect of adopting a primary nitrogen contribution from massive stars.

Results. The $[\alpha / \mathrm{Fe}]$ abundance ratios in the Bulge are predicted to be supersolar for a very large range in $[\mathrm{Fe} / \mathrm{H}]$, each element having a different slope. These predictions are in very good agreement with most recent accurate abundance determinations. We also find a good fit of the most recent Bulge stellar metallicity distributions.

Conclusions. We conclude that the Bulge formed on a very short timescale (even though timescales much shorter than $\sim 0.1 \mathrm{Gyr}$ are excluded) with a quite high star formation efficiency of $v \simeq 20 \mathrm{Gyr}^{-1}$ and with an initial mass function more skewed toward high masses (i.e. $x \leq 0.95$ ) than the solar neighbourhood and rest of the disk. The results obtained here are more robust than previous ones since they are based on very accurate abundance measurements.
\end{abstract}

Key words. Galaxy: bulge - Galaxy: evolution - Galaxy: abundances - Galaxy: formation

\section{Introduction}

Galactic bulges are spheroidal systems that are found in the centre of most spiral galaxies, and usually possess metallicity, photometric and kinematic properties that separate them from the disk components. Studying the evolution of the bulge of the Galaxy is of general interest because of the broad similarity of its integrated light to elliptical galaxies and other spiral bulges (e.g. Whitford 1978; Maraston et al. 2003). Bulges and ellipticals are located in the same regions of the fundamental plane (Jablonka et al. 1996); the Galactic bulge has a wide range in [Fe/H] (Rich 1988; McWilliam \& Rich 2004; Zoccali et al. 2003; Fulbright et al. 2005) and enhancement of $\alpha$-elements (McWilliam \& Rich 1994; McWilliam \& Rich 2004; Rich \& Origlia 2005); the age dispersion in the bulge at distances greater than $300 \mathrm{pc}$ from the nucleus is observed to be narrow. Terndrup (1988) first argued for a globular cluster-like nature to the bulge population. Ortolani et al. (1995) compared the luminosity function of a metal rich globular cluster with that of the bulge field Baade's Window. Recently, Kuijken \& Rich (2002) and Zoccali et al. (2003) showed that when the bulge field is decontaminated from disk foreground stars by proper motion cleaning or statistical subtraction, the remaining population is indistinguishable from an old metal rich globular cluster. These studies form the basis for a growing consensus that the bulge is old and that its formation timescale was relatively short ( $\leq 1 \mathrm{Gyr}$ ). Very recently, medium- and high-resolution spectroscopy of bulge stars have been performed (Ramírez et al. 2000; Fulbright et al. 2006a, FMR06a), providing further support to a fast formation of the bulge.

Finally, a short formation timescale for the bulge is also suggested on theoretical grounds by Elmegreen (1999), who argued that the potential well of the Galactic bulge is too deep to allow self-regulation and that most of the gas must have been converted into stars within a few dynamical timescales. Moreover, Sarajedini \& Jablonka (2005) suggest that, since the differences in the metallicity distributions (MDs) of the Milky Way and M 31 halos find no correspondence in those of their bulges, the bulk of the stars in the bulges of both galaxies must have been in place before any accretion event that might have occurred in the halo could have any influence on them, supporting a common scenario for the formation of bulges.

Wyse (1998) also showed that the MD in our Galaxy is not consistent with a picture where the bulge is formed via accretion of satellites (see Searle \& Zinn 1978). There is now additional evidence that the bulge is not formed by satellites similar to those observed at the present day in the halos of the Milky Way and M31. McWilliam et al. (2003) find a systematically decreased abundance of $\mathrm{Mn}$ in the Galactic bulge, compared to stars in the Sgr dwarf spheroidal. So, even though Sgr does in principle reach high metallicity, its detailed chemistry is different.

The baseline model for the chemical evolution of the bulge might well be the one-zone model (Searle \& Zinn 1978) with its instantaneous recycling and closed-box assumptions. Early 
numerical models (Arimoto \& Yoshii 1986) produced the observed wide abundance range. When supernova yields and detailed stellar lifetimes are incorporated, more extensive predictions become possible. This is the case of the Galactic bulge model by Matteucci \& Brocato (1990, MB90) who predicted that the $[\alpha / \mathrm{Fe}]$ ratio for some elements $(\mathrm{O}, \mathrm{Si}$ and $\mathrm{Mg})$ should be supersolar over almost the whole metallicity range, in analogy with the halo stars, as a consequence of assuming a fast bulge evolution which involved rapid gas enrichment in Fe mainly by Type II SNe. At that time, no data were available for detailed chemical abundances; the predictions of MB90 were later confirmed for a few $\alpha$-elements (Mg, Ti) by the observations of McWilliam \& Rich (1994, MR94). A few years later, Matteucci et al. (1999, MRM99) modeled the behaviour of a larger set of abundance ratios, by means of a detailed chemical evolution model whose parameters were calibrated so that the metallicity distribution observed by MR94 could be fit. They predicted the evolution of several abundance ratios that were meant to be confirmed or disproved by subsequent observations, namely that $\alpha$-elements should in general be overabundant with respect to $\mathrm{Fe}$, but some (e.g. Si, Ca) less than other (e.g. $\mathrm{O}, \mathrm{Mg}$ ), and that the $\left[{ }^{12} \mathrm{C} / \mathrm{Fe}\right]$ ratio should be solar at all metallicities. They concluded that an IMF index flatter $(x=1.1-1.35)$ than that of the solar neighbourhood is needed for the MR94 metallicity distribution to be reproduced, and that an evolution much faster than that in the solar neighbourhood and faster than that of the halo (see also Renzini 1993) is necessary as well. Whatever secular processes may be at work with respect to the formation and maintenance of the bar, the chemistry and stellar ages require that the stars formed early and self-enriched rapidly.

Not all models of the bulge support these conclusions. Samland et al. (1997) developed a self-consistent chemodynamical model for the evolution of the Milky Way components starting from a rotating protogalactic gas cloud in virial equilibrium, that collapses owing to dissipative cloud-cloud collisions. They found that self-regulation due to a bursting star formation and subsequent injection of energy from Type II supernovae led to the development of "contrary flows", i.e. alternate collapse and outflow episodes in the bulge. This caused a prolonged star formation episode lasting over $\sim 4 \times 10^{9} \mathrm{yr}$. They included stellar nucleosynthesis of $\mathrm{O}, \mathrm{N}$ and $\mathrm{Fe}$, but claim that gas outflows prevent any clear correlation between local star formation rate and chemical enrichment. With their model, they could reproduce the oxygen gradient of $\mathrm{H}$ II regions in the equatorial plane of the Galactic disk and the metallicity distribution of $\mathrm{K}$ giants in the bulge (Rich 1988), field stars in the halo and G dwarfs in the disk, but they did not make predictions about the evolution of abundance ratios in the bulge.

Mollá et al. (2000) proposed a multiphase model in the context of the dissipative collapse scenario of the Eggen et al. (1962) picture. They suppose that the bulge formation occurred in two main infall episodes, the first from the halo to the bulge, on a timescale $\tau_{\mathrm{H}}=0.7 \mathrm{Gyr}$ (longer than that proposed by MRM99), and the second from the bulge to a so-called core population in the very nuclear region of the Galaxy, on a timescale $\tau_{\mathrm{B}} \gg \tau_{\mathrm{H}}$. The three zones (halo, bulge, core) interact via supernova winds and gas infall. They concluded that there is no need for accretion of external material to reproduce the main properties of bulges and that the analogy to ellipticals is not justified. Because of their rather long timescale for the bulge formation, these authors did not predict a noticeable difference in the trend of the $[\alpha / \mathrm{Fe}]$ ratios but rather suggested that they behave more likely as in the solar neighbourhood (contrary to the first indications of $\alpha$-enhancement by MR94).
A more recent model was proposed by Costa et al. (2005), in which the best fit to the observations relative to planetary nebulae (PNe) is achieved by means of a double infall model. An initial fast $(0.1 \mathrm{Gyr})$ collapse of primordial gas is followed by a supernova-driven mass loss and then by a second, slower ( 2 Gyr) infall episode, enriched by the material ejected by the bulge during the first collapse. Costa et al. (2005) claim that the mass loss is necessary to reproduce the abundance distribution observed in PNe, and because the predicted abundances would otherwise be higher than observed. With their model, they are able to reproduce the trend of $[\mathrm{O} / \mathrm{Fe}]$ abundance ratio observed by Pompéia et al. (2003) and the data of nitrogen versus oxygen abundance observed by Escudero \& Costa (2001) and Escudero et al. (2004). It must be noted however that Pompéia et al. (2003) obtained abundances for "bulge-like" dwarf stars. This "bulgelike" population consists of old ( $10-11 \mathrm{Gyr})$, metal-rich nearby dwarfs with kinematics and metallicity suggesting an inner disk or bulge origin and a mechanism of radial migration, perhaps caused by the action of a Galactic bar. However, the birthplace of these stars is not certain, therefore we decided to omit these data from our model discussions, preferring to consider those stars for which membership in the present day bulge is secure. Moreover, as we shall see, the use of nitrogen abundance from $\mathrm{PNe}$ is questionable, since $\mathrm{N}$ is known to be also synthesized by their progenitors and therefore it might not be the pristine one.

In this paper we want to test the hypothesis of a quick dissipational collapse via the study of the evolution of the abundance ratios coupled with considerations on the metallicity distribution. Ferreras et al. (2003) already tried to fit the stellar metallicity distributions of K giants of Sadler et al. (1996), Ibata \& Gilmore (1995) and Zoccali et al. (2003), which are pertinent to different bulge fields, by means of a model of star formation and chemical evolution. Their model assumes a Schmidt law similar to that of the disk, and simple recipes with a few parameters controlling infall and continuous outflow of gas. They explore a large range in parameter space and conclude that timescales longer than $\sim 1$ Gyr must be excluded at the $90 \%$ confidence level, regardless of which field is being considered. We want to show that abundance ratios can provide an independent constraint for the bulge formation scenario since they differ depending on the star formation history (Matteucci 2000).

We focus our attention on the evolution of the $\alpha$-elements, carbon and nitrogen as a function of metallicity. New data have lately become available for the abundance ratios of these nuclids: $\alpha$-elements in particular are of paramount importance in probing the star formation timescale, since the signature of a very short burst of star formation must result into an $\alpha$-enhancement with respect to iron, whereas $\sim$ solar abundances would imply that Type Ia Supernovae ( $\mathrm{SNe}$ ) had time to pollute the ISM with iron-rich ejecta (see e.g. Matteucci \& Greggio 1986). Moreover, we also analysed the evolution of nitrogen and studied the possibility of primary $\mathrm{N}$ being produced by all massive stars at any metallicity, as described in Matteucci (1986). This seems to provide a good fit to $[\mathrm{N} / \mathrm{O}]$ and $[\mathrm{N} / \mathrm{Fe}]$ in the solar vicinity (Chiappini et al. 2005; Ballero et al. 2006; see also Hirschi 2006). The present paper does not primarily mean to point at a particular model as the "best model", but rather has the aim of showing which are the possible effects of varying the abovementioned parameters; in fact, since the results for the MD of bulge giants are still preliminary, and high-resolution data are awaited, it is premature to draw firm conclusions on which combination of parameters provides the best fit. However, we can quite safely restrict the ensemble of plausible models by means of the present analysis. 
The paper is organized as follows: Sect. 2 describes the chemical evolution model, Sect. 3 provides a review of the data employed, Sect. 4 shows the model results regarding the supernova rates, the chemical evolution of abundance ratios, the MD of $\mathrm{G}, \mathrm{K}$ and $\mathrm{M}$ giants in the bulge and in Sect. 5 we draw our conclusions.

\section{The chemical evolution models}

The adopted basic chemical evolution model closely follows that in MRM99. The main assumption is that the Galactic bulge formed with the fast collapse of primordial gas (the same gas out of which the halo was formed) accumulating in the centre of our Galaxy. We recall the fundamental ingredients of this model:

- Instantaneous mixing approximation: the gas over the whole bulge is homogeneous and well mixed at any time.

- Star formation rate (SFR) parametrized as follows:

$$
\psi(r, t)=v G^{k}(r, t)
$$

where $v$ is the star formation efficiency (i.e. the inverse of the timescale of star formation) in the bulge, $k=1$ is chosen to recover the star formation law employed in models of spheroids (e.g. by Matteucci 1992) and $G(r, t)=$ $\sigma_{\text {gas }}(r, t) / \sigma\left(r, t_{G}\right)$ is the normalized gas surface mass density (where $\sigma_{\text {gas }}(r, t)$ is the gas surface mass density and $\sigma\left(r, t_{G}\right)$ is the surface gas density of the bulge at the present time $t_{G}=13.7 \mathrm{Gyr}$ ). We tested also different values for $k$ (such as 1.5 , which is the disk value) and the result do not differ much. The main difference with the solar neighbourhood resides in the higher $v$ parameter for the bulge.

- We did not adopt a thresold surface gas density for the onset of star formation such as that proposed by Kennicutt (1998) for the solar neighbourhood, since it is derived for self-regulated disks and there seems to be no reason for it to hold also in early galaxy evolutionary conditions and in bulges. However, we also checked that adopting a threshold of $4 M_{\odot} \mathrm{pc}^{-2}$ such as that proposed by Elmegreen (1999) does not change our results, since a wind (see below) develops much before such a low gas density is reached.

- The initial mass function (IMF) is expressed as a power law with index $x$ :

$\phi(m) \propto m^{-(1+x)}$

within the mass range $0.1-100 M_{\odot}$.

- The gas forming the bulge has a primordial chemical composition and accretes at a rate given by:

$\dot{G}(r, t)_{\text {inf }}=\frac{A(r)}{\sigma\left(r, t_{G}\right)} \mathrm{e}^{-t / \tau}$

where $\tau$ is an appropriate collapse timescale and $A(r)$ is constrained by the requirement of reproducing the current total surface mass density in the Galactic bulge. Actually, we should use the halo chemical composition for the infalling gas, but it can be demonstrated that unless very high $\alpha$-enhancements are adopted, the results are essentially the same. In principle, a slightly enriched infall could improve the fit of the MDs (see later) but this is not likely to have significant effects for realistic levels of enrichment.

- The instantaneous recycling approximation is relaxed; stellar lifetimes are taken into account in detail following the prescriptions of Kodama (1997).
- Detailed nucleosynthesis prescriptions are taken from François et al. (2004), who made use of widely adopted stellar yields and compared the results obtained by including these yields in the model from Chiappini et al. (2003) with the observational data, with the aim of constraining the stellar nucleosynthesis. Namely, for low- and intermediatemass $\left(0.8-8 M_{\odot}\right)$ stars, which produce ${ }^{12} \mathrm{C}, \mathrm{N}$ and heavy $s$-elements, yields are taken from the standard model of van den Hoek \& Groenewegen (1997) as a function of the initial stellar metallicity. Concerning massive stars $(M>$ $\left.10 M_{\odot}\right)$, in order to best fit the data in the solar neighbourhood, when adopting Woosley \& Weaver (1995) ${ }^{1}$ yields, François et al. (2004) found that $\mathrm{O}$ yields should be adopted as a function of initial metallicity, $\mathrm{Mg}$ yields should be increased in stars with masses $11-20 M_{\odot}$ and decreased in stars larger than $20 M_{\odot}$, and that $\mathrm{Si}$ yields should be slightly increased in stars above $40 M_{\odot}$; we use their constraints on the stellar nucleosynthesis to test whether the same prescriptions give good results for the Galactic bulge. Yields in the mass range $40-100 M_{\odot}$ were not computed by Woosley and Weaver (1995), therefore one has to extrapolate them for chemical evolution purposes ${ }^{2}$.

- The Type Ia SN rate was computed according to Greggio \& Renzini (1983) and Matteucci \& Recchi (2001). Yields are taken from Iwamoto et al. (1999) which is an updated version of model W7 (single degenerate) from Nomoto et al. (1984). These supernovae are the main contributors of Fe and produce small amounts of light elements; they also contribute to some extent to the enrichment in $\mathrm{Si}$ and $\mathrm{Ca}$.

- Contrary to MRM99, we introduced the treatment of a supernova-driven galactic wind in analogy with ellipticals (e.g. Matteucci 1994). The bulge lies in the potential well of the Galactic disk and very massive dark halo that provides a high binding energy. Therefore, previous chemical evolution models supposed that a wind should not develop and moreover, the occurrence of winds did not seem to be suitable to reproduce the metallicity distribution of the Milky Way components (Tosi et al. 1998), especially for the disk. Moreover, Elmegreen (1999) sustained that the bulge potential well is too deep to allow for self-regulation and that the gas should be converted into stars in only a few dynamical timescales. However, this scenario was not tested quantitatively. We therefore supposed that the bulge is bathed in a dark matter halo of mass 100 times greater than that of the bulge itself (i.e. $M_{\text {dark }}=2 \times 10^{12} M_{\odot}$ ) and with an effective radius $r_{\text {dark }}=100 r_{\mathrm{e}}=200 \mathrm{kpc}$, where $r_{\mathrm{e}}$ is the effective radius of the bulge (Sérsic) mass distribution. To compute the gas binding energy $E_{\mathrm{b}, \mathrm{gas}}(t)$ we have followed Matteucci (1992) who adopted the formulation of Bertin et al. (1992). They

1 The models of Woosley \& Weaver (1995) do not include mass loss, which might not be realistic for non-zero metallicity stars. However, it was shown (Bressan et al. 1981; Bertelli et al. 1990) that the combined effects of mass loss and overshooting compensate each other, in the sense that the resulting He-core mass (where all the heavy elements are produced) remains approximately the same as that in models without mass loss and overshooting. Additionally, nucleosynthesis with mass loss and rotation has so far been computed only for a few elements (e.g. Maeder et al. 2005).

2 The authors are aware that the extrapolation process is problematic. However, the behaviour above this mass is not clear, since a supernova explosion may occur with a large amount of fallback. Moreover, it was shown (François et al. 2004) that it is impossible to reproduce the observations at low metallicities in the solar neighbourhood if no contribution from stars in this mass range is considered. 
analysed the properties of a family of self-consistent spherical two-component models of elliptical galaxies, where the luminous mass is embedded in massive and diffuse dark halos, and in this context they computed binding energy of the gas. A more refined treatment of the Galactic potential well would take into account also the contribution of the disk; however, it is easy to show that the main contributors to the bulge potential well are the bulge itself and the dark matter halo. The condition for the onset of the galactic wind is:

$$
E_{\mathrm{th}, \mathrm{SN}}\left(t_{\mathrm{GW}}\right)=E_{\mathrm{b}, \mathrm{gas}}\left(t_{\mathrm{GW}}\right)
$$

where $E_{\mathrm{th}, \mathrm{SN}}(t)$ is the thermal energy of the gas at the time $t$ owing to the energy deposited by $\mathrm{SNe}$ (II and Ia) (see Matteucci 1992 and Bradamante et al. 1998). At the specific time $t_{\mathrm{GW}}$ (the time for the occurrence of a galactic wind), all the remaining gas is expelled from the bulge, and both star formation and gas infall cease. However, in all cases, the galactic wind occurs when most of the gas has already been converted into stars, and its effect on chemical evolution is negligible. Therefore, we shall limit ourselves to switching off star formation and infall when $t_{\mathrm{GW}}$ is reached.

- We suppose that feedback from the central black hole is negligible. We shall test this hypothesis quantitatively in a forthcoming paper.

As a fiducial model, we adopt the one with the following reference parameters: $v=20 \mathrm{Gyr}^{-1}$, collapse timescale $\tau=0.1 \mathrm{Gyr}$ and a two-slope IMF with index $x=0.33$ for $M<1 M_{\odot}$ and $x=0.95$ for $M>1 M_{\odot}$ (Matteucci \& Tornambè 1987). The choice of such a flat IMF for the lowest-mass stars is motivated by the Zoccali et al. (2000) work, who measured the luminosity function of lower main-sequence bulge stars and derived the mass function, which was found to be consistent with a power-law of index $0.33 \pm 0.07$. The IMF index for intermediatemass and massive stars which is slightly flatter than that adopted by MRM99 in order to reproduce the MD of bulge stars from Zoccali et al. (2003, Z03) and Fulbright et al. (2006a, FMR06a; see Sects. 3 and 4.2 for details) instead ot that from MR94. We explored a number of other possibilities by varying the model parameters in the following way:

- star formation efficiency: $v$ from 2 to $200 \mathrm{Gyr}^{-1}$;

- for the IMF above $1 M_{\odot}$, we have considered the cases suggested by Zoccali et al. (2000, Z00) in their Sect. 8.3, i.e. their case 1 (hereafter Z00-1) with $x=0.33$ in the whole range of masses, their case 3 (Z00-3) for which $x=1.35$ for $M>1 M_{\odot}$ (Salpeter 1955) and their case 4 (Z00-4) in which $x=1.35$ for $1 M_{\odot}<M<2 M_{\odot}$ and $x=1.7$ for $M>2 M_{\odot}$ (Scalo 1986). Our reference model corresponds to their case 2 with $x=0.95$ for $M>1 M_{\odot}{ }^{3}$, therefore we call it Z00-2. We recall that the fraction $A$ of binary systems giving rise to Type Ia $\mathrm{SNe}$ is a function of the adopted IMF (see Matteucci \& Greggio 1986). Owing to the lack of information concerning the Type Ia SN rate in the bulge, we calibrate such a fraction in order to reproduce the Mannucci et al. (2005) estimate of the SN rate of an elliptical galaxy of the same mass;

- infall timescale: $\tau$ from 0.01 to $0.7 \mathrm{Gyr}$; the latest hypothesis follows the suggestion by Mollá et al. (2000) who assume a slower timescale for the formation of the bulge. We refer to the timescale $\tau_{\mathrm{H}}$ which they chose for the gas collapse from halo to bulge.

\footnotetext{
3 Actually, Z00 selected as "IMF 2" the one with $x=1$ for $M>1 M_{\odot}$, but we performed calculations with $x=0.95$, which is very similar, for comparison to the IMF with $x=0.95$ of (Matteucci \& Tornambè 1987).
}

Table 1. Features of the examined models.

\begin{tabular}{llcl}
\hline \hline Model name/specification & $x\left(M>1 M_{\odot}\right)$ & $v\left(\mathrm{Gyr}^{-1}\right)$ & $\tau(\mathrm{Gyr})$ \\
\hline Fiducial model $($ Z00-2) & 0.95 & 20.0 & 0.1 \\
\hline Z00-1 & 0.33 & 20.0 & 0.1 \\
Z00-3 & 1.35 & 20.0 & 0.1 \\
Z00-4 & Scalo* $^{*}$ & 20.0 & 0.1 \\
\hline$v=2 \mathrm{Gyr}^{-1}$ & 0.95 & 2.0 & 0.1 \\
$v=200 \mathrm{Gyr}^{-1}$ & 0.95 & 200.0 & 0.1 \\
\hline$\tau=0.01 \mathrm{Gyr}$ & 0.95 & 20.0 & 0.01 \\
$\tau=0.7 \mathrm{Gyr}$ & 0.95 & 20.0 & 0.7 \\
\hline S1 & 0.33 & 200.0 & 0.01 \\
S2 & 0.95 & 200.0 & 0.01 \\
S3 & 0.33 & 2.0 & 0.7 \\
S4 & 0.95 & 2.0 & 0.7 \\
S5 & 0.33 & 0.5 & 1.25 \\
\hline
\end{tabular}

Table 1 summarizes the features of the considered models.

\section{The data}

We now turn to the datasets against which the models will be compared. These are re-normalized to the solar abundances of Grevesse \& Sauval (1998) so that an artificial dispersion associated with the adoption of different solar abundance values is corrected for.

\section{Metallicity distribution}

Data for the $[\mathrm{Fe} / \mathrm{H}]$ distribution of red giant branch $(\mathrm{RGB})$ and asymptotic giant branch (AGB) stars in the bulge were taken from $\mathrm{Z} 03$, who provided photometric determination of metallicities for 503 bulge stars. By combining near-infrared data from the 2MASS survey, from the SOFI imager at ESO NTT and the NICMOS camera on HST, plus optical images taken with the WFI at ESO/MPG $2.2 \mathrm{~m}$ telescope within the EIS PRE-FLAMES survey, they constructed a disk-decontaminated $\left(M_{K}, V-K\right)$ color-magnitude diagram (CMD) of the bulge stellar population with very large statistics and small photometric errors, which was compared with the analytical RGB templates in order to derive the MD. The advantage of this approach is that it allows determinations of metallicities of a great number of stars, although the relationship between the position in the CMD and the metallicity can be somewhat uncertain.

Since the template RGBs are on the $[\mathrm{M} / \mathrm{H}]$ scale (where M stands for the total metal abundance), in order to obtain the $[\mathrm{Fe} / \mathrm{H}]$ distribution the $\alpha$-enhancement contribution was subtracted in the following way (Zoccali, private communication):

$[\mathrm{Fe} / \mathrm{H}]= \begin{cases}{[\mathrm{M} / \mathrm{H}]-0.14} & \text { if }[\mathrm{M} / \mathrm{H}]>-0.86 \\ {[\mathrm{M} / \mathrm{H}]-0.21} & \text { if }[\mathrm{M} / \mathrm{H}]<-0.86\end{cases}$

This relation assumes that the $\alpha$-elements in the bulge follow the abundance trends of globular clusters in the halo. They found that the resulting MD contained somewhat less metalpoor stars relative to a closed-box gas exhaustion model and that the G-dwarf problem (i.e. the deficit of metal-poor stars relative to a simple model) may affect the Galactic bulge even though less severely than in the solar neighbourhood (see e.g. Hou et al. 1998).

In Fig. 1 we compare the photometric MD derived by Z03 to the spectroscopic one of MR94. The two distributions are broadly consistent, with a somewhat less pronounced supersolar $[\mathrm{Fe} / \mathrm{H}]$ tail and a slightly sharper peak in the photometric case. 


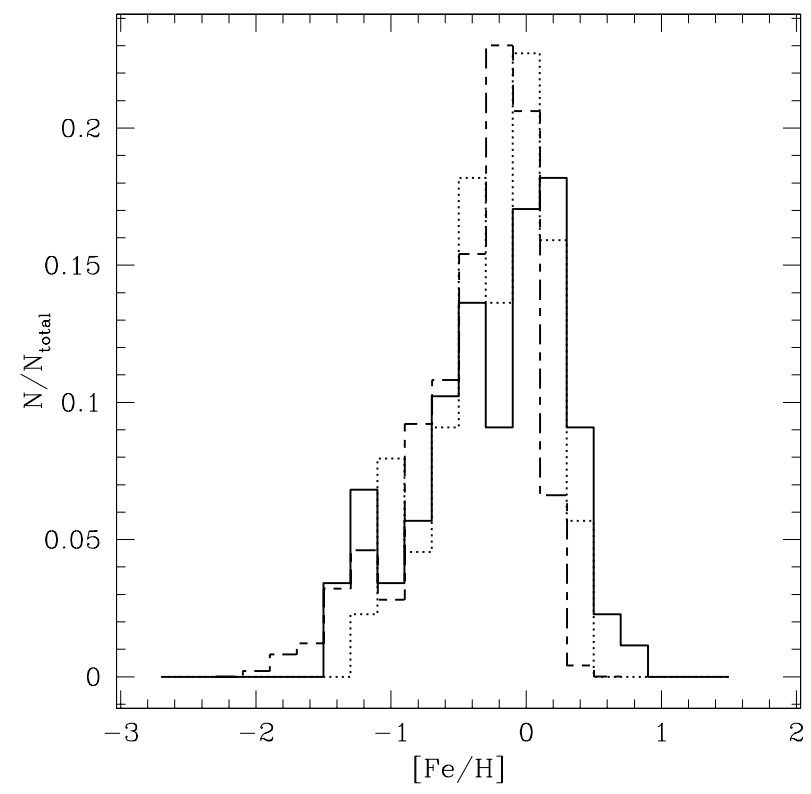

Fig. 1. Comparison between the bulge MD derived by Z03 (solid histogram), FMR06a (dashed histogram) and that derived with the spectroscopic survey of MR94 (dotted histogram).

However, the position of the high $[\mathrm{Fe} / \mathrm{H}]$ cutoff for the photometric $\mathrm{MD}$ is wholly dependent on the metallicity assigned to the only two template clusters, namely NGC 6528 and NGC 6553 available in the high-metallicity domain. Nevertheless, there are also indications (Zoccali, private communication) that the MD of the bulge stars, when achieved with high-resolution spectroscopy, is comparable to or narrower than that showed here, and that there are a very few supersolar-metallicity stars.

In the same figure we also show the data from FMR06a, who performed a new high-resolution $(R \sim 45000-60000)$ analysis of $27 \mathrm{~K}$-giants in the Baade's Window $\left(b=-4^{\circ}\right)$ sample with the HIRES spectrograph on the Keck I telescope and, after determining their $\mathrm{Fe}$ abundances, they used them as reference stars in order to re-calibrate the K-giant data from Rich (1988). They found that the derived MDs are slightly stretched toward both the metal-poor and metal-rich tails with respect to those derived in previous works (Rich 1988; MR94; Sadler et al. 1996; Zoccali et al. 2003), although the overall consistency among these different MDs is reasonable. The metal-rich end probably extends to spuriously high abundances.

\section{$\alpha$-element and carbon abundances}

- Abundances of $\mathrm{O}, \mathrm{Mg}, \mathrm{Si}, \mathrm{Ca}, \mathrm{C}$ and $\mathrm{Fe}$ for stars and clusters in the bulge are taken from Origlia et al. (2002) Origlia \& Rich (2004), Origlia et al. (2005) and Rich \& Origlia (2005). We refer to these abundances as the "IR spectroscopic database" hereafter. These datasets were obtained using the NIRSPEC spectrograph at Keck II, which allowed the determination of near-infrared, high-resolution $(R \sim 25000)$, high signal-to-noise ratio $(S / N>40)$ échelle spectra and were the only ones to provide $C$ abundances for bulge giants. They used the $1.6 \mu \mathrm{m}$ region of the spectrum, corresponding to the $\mathrm{H}$ band. In all cases, abundance analysis was performed by means of full spectrum synthesis and equivalent width measurements of representative lines. Reliable oxygen abundances were derived from a number of $\mathrm{OH}$ lines; similarly, the $\mathrm{C}$ abundance was derived from
$\mathrm{CO}$ molecular lines, whereas strong atomic lines were measured for $\mathrm{Mg}, \mathrm{Si}, \mathrm{Ca}, \mathrm{Ti}$ and $\mathrm{Fe}$. The data include observations of bright giants in the cores of the bulge globular clusters Liller 1 and NGC 6553 (Origlia et al. 2002; see also Melèndez et al. 2003), Terzan 4 and Terzan 5 (Origlia \& Rich 2004), NGC 6342 and NGC 6528 (Origlia et al. 2005, see also Carretta et al. 2001; Zoccali et al. 2004) and measurements of abundances of $\mathrm{M}$ giants in Baade's window (Rich \& Origlia 2005). The typical errors are of $\pm 0.1 \mathrm{dex}$. The main considerations that were drawn from these abundance analyses are that $\alpha$-enhancement is safely determined in old stars with $[\mathrm{Fe} / \mathrm{H}]$ as high as solar, pointing toward early formation and rapid enrichment in both clusters and field, which are likely to share a common formation history. The $[\mathrm{C} / \mathrm{Fe}]$ abundance ratios can be depleted up to a factor of $\approx 3$ with respect to the solar value, as expected because of the first dredge-up and possibly extra-mixing mechanisms due to cool bottom processing, which are at work during the evolution along the RGB, as also indicated by the very low $(<10){ }^{12} \mathrm{C} /{ }^{13} \mathrm{C}$ abundance ratio (see also Origlia et al. 2003 and references therein). The analysis of $M$ giants yielded abundances similar to those obtained with high-resolution optical spectroscopy of K giants; there is an apparent lack of supersolar- $[\mathrm{Fe} / \mathrm{H}]$ stars, but the sample is too small to draw firm conclusions.

- For $\mathrm{O}, \mathrm{Mg}, \mathrm{Si}$ and $\mathrm{Ca}$ we also included the abundance measurements of Fulbright et al. (2006b, FMR06b), who used the same spectra as in FMR06a, i.e. obtained the spectra of 27 bulge $\mathrm{K}$ giant stars at the Keck I telescope using the HIRES échelle spectrograph with high resolution $(R \sim 45000-67000)$ and high signal-to-noise ratio. The typical errorbars are of $\sim 0.1$ dex. The outcome of their analysis is that all elements produced from massive stars (i.e. $\alpha$-elements, plus $\mathrm{Na}$ and $\mathrm{Al}$ ) show enhancement in bulge stars relative to both Galctic thick and thin disk, although oxygen shows a sharply decreasing trend for supersolar $[\mathrm{Fe} / \mathrm{H}]$, which is attributed to a metallicity-dependent modulation of the oxygen yield from massive stars. These results suggest that massive stars contributed more to the chemical enrichment of the bulge than to the disk, and consequently that the timescale for bulge formation were shorter than that of the disk, although they did not exclude other possibilities (such as, e.g., an IMF skewed to high masses).

- Finally, oxygen data from Zoccali et al. (2006, Z06) were also taken. In this paper, $\mathrm{Fe}$ and $\mathrm{O}$ abundances for $50 \mathrm{~K}$ giants in four fields $(b=-6$; Baade's Window; Blanco $b=-12$; NGC 6553) towards the Galactic bulge were derived; oxygen abundance was measured from the forbidden line at $6300 \AA$. A high resolution $(R=45000)$ was achieved with FLAMES-UVES at the VLT. The typical errorbars are of $\sim 0.1 \mathrm{dex}$. Also in this case, $[\mathrm{O} / \mathrm{Fe}]$ is found to be higher in bulge stars than both in thick and thin disk, and supports a scenario where the bulge formed before the disk and more rapidly, with a formation history similar to that of old earlytype galaxies.

\section{$\mathrm{N}$ vs. $\mathrm{O}$}

Our nitrogen abundances in the bulge are derived from PNe. This may represent a problem, since while the measured oxygen abundance represents the true value of the ISM out of which the PN progenitor was formed, the observed $\mathrm{N}$ abundance has contributions both from the pre-existent nitrogen and from that 

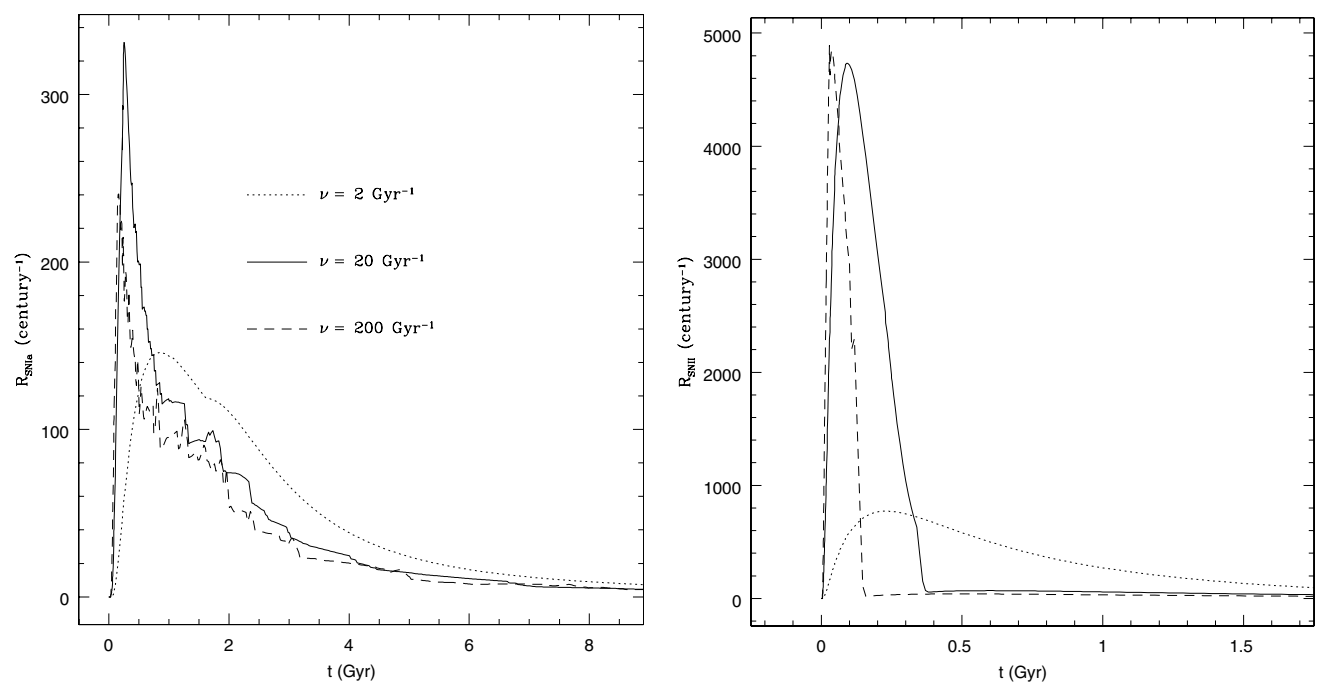

Fig. 2. Evolution of the rates of type Ia (left) and type II (right) supernovae where different values of the star formation efficiency (middle panel).

produced by the star itself during its lifetime and dredged-up to the stellar surface. Moreover, these data come from emission lines which have a very complicated dependence upon several parameters (such as temperature, density and metal content) and assumptions (e.g. the photoionization model, from where the largest uncertainties come). Therefore it might be dangerous to employ these measurements to trace galactic chemical evolution. In principle, it would be possible to discriminate between enriched and primordial $\mathrm{N}$ by means of the $\mathrm{C} / \mathrm{N}$ ratio (which is very different in the two cases, being much lower in the case of nitrogen-enriched stars). Unfortunately, measurements for carbon in bulge PNe are available to date only for a very limited set of objects (Webster 1984; Walton et al. Walton; Liu et al. 2001). Another possibility is to make use of symbiotic stars, i.e. presumably $\mathbf{M}$ giants in binary systems with a white dwarf or another hot companion. The envelope of the symbiotic star is photoionized from the hard UV radiation, leading to recombination line emission. Since the envelope is being observed, the abundances may be less evolved than in the PNe (Nussbaumer et al. 1988).

It is possible that the $\mathrm{N}$ enrichment is not very dramatic especially in non-Type I PNe, which constitute about $80 \%$ of the PN population in the Galaxy (Peimbert \& Serrano 1980). Moreover, since the bulge presumably has not formed stars for a long time, Type I PNe (which have high-mass progenitors and are the most nitrogen-enriched) are not expected to be frequent. This is also confirmed by Cuisinier et al. (2000) who found quite low [N/O] ratios in their sample if compared to that resulting from selfenrichment. Moreover, Luna \& Costa (2005) measured the N/O ratio of 43 symbiotic stars towards the Galactic bulge and, as can be seen in their Fig. 5, the values of $\log (\mathrm{N} / \mathrm{O})$ are consistent with those coming from studies of PNe.

We employed the compilation of Górny et al. (2004), who observed $44 \mathrm{PNe}$ in the direction of the Galactic bulge with the aim of discovering Wolf-Rayet stars at their centre. The spectra were obtained with the 1.9-m telescope at the South African Astronomical Observatory, with an average resolution of 1000. Furthermore, Górny et al. (2004) also merged their data with other published ones. Namely, they included the samples observed by Cuisinier et al. (2000), Escudero \& Costa (2001) and Escudero et al. (2004). They obtained a total of 164 objects, among which a clear segregation of the subsamples is seen, due to the different selection criteria adopted to define each sample, and therefore none of them is truly representative of the bulge PN population. By merging the datasets, a more complete view of this population is achieved. Updated reddening corrections were applied to the objects from Escudero \& Costa (2001) and Escudero et al. (2004). The merged sample was divided into two classes (according to the criteria listed in Stasińska et al. 1991), the first including those objects which are likely to be physically related to the Galactic bulge and the second containing the remaining objects which most probably belong to the disk. We only selected objects belonging to the bulge which had a clear detection of oxygen and nitrogen emission features; the resulting sample includes 103 objects. Errors in abundance derivations from both observational and theoretical uncertainties are typically $0.2-0.3$ dex for $[\mathrm{O} / \mathrm{H}]$ and can be even larger for $[\mathrm{N} / \mathrm{O}]$.

In the future, when very high resolution IR spectroscopy ( $R \approx 100000$ ) becomes possible, $\mathrm{N}$ estimates could be also derived in giant stars from the faint $\mathrm{CN}$ lines that are de-blended from stronger the $\mathrm{CO}$ and $\mathrm{OH}$ lines at high resolution.

\section{Model results}

\subsection{Supernova rates}

Figure 2 shows the predicted time evolution of the rate of type II and Type Ia SNe in the Galactic bulge; the former, that die on small timescales, closely reflect the evolution of the SFR (for simplicity, we only show the case of different star formation efficiencies). The secondary peaks of the SNIa rates are softwarerelated and are mostly due to the discontinuities in the adopted stellar lifetimes (Kodama 1997) but do not affect the results concerning chemical abundances. The break in the Type II SN rates corresponds to the suppression of the star formation rate due to the achievent of the condition expressed in Eq. (2), which quite intuitively occurs earlier for flatter IMFs, higher $v$ 's and/or lower $\tau$ 's. However, even without a galactic wind, the Type II SN rate would become negligible at the same epoch, owing to the small amount of gas left in the bulge at that time.

In those cases where the star formation is "bursty" (e.g. high star formation efficiency and quick formation timescale), the peak of the Type Ia SN rate (and therefore of Fe enrichment) can occur even before $1 \mathrm{Gyr}$, which is the timescale for Fe-enrichment in the solar neighbourhood. In fact, the time of occurrence of this peak is very sensitive to the underlying star 

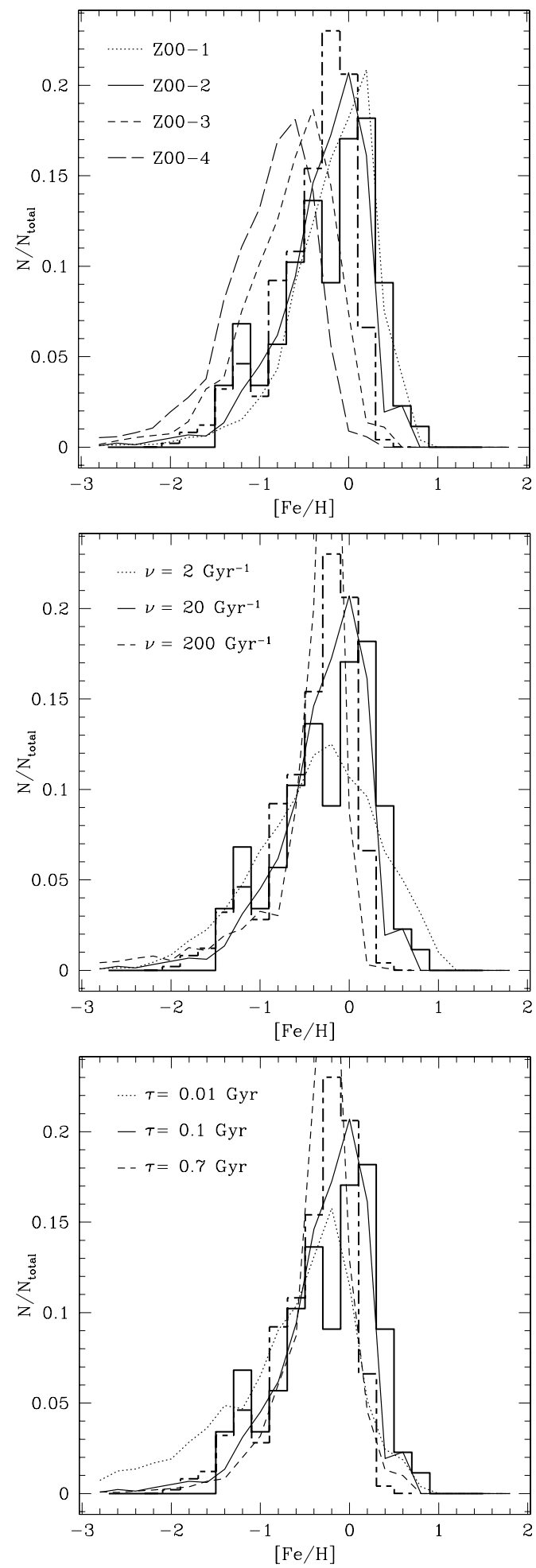

Fig. 3. Predicted metallicity distributions in our bulge models for single parameter variations relative to the reference model, with different choices of the IMF index (upper panel; see text for details), star formation efficiency (middle panel), and gas infall timescale (lower panel). Our fiducial model is represented by the solid line. Data are from Z03 (solid histogram) and FMR06a (dashed histogram).

formation history and can be as low as $\sim 0.2$ Gyr in the case of $\tau=0.01 \mathrm{Gyr}$ or $v=200 \mathrm{Gyr}^{-1}$ (see also Matteucci \& Recchi 2001). Therefore, we expect that:

1. The down-turn (i.e. change of slope) of the $[\alpha / \mathrm{Fe}]$ vs. $[\mathrm{Fe} / \mathrm{H}]$ plots, corresponding to the beginning of a sustantial $\mathrm{Fe}$ enrichment, will occur later, in general, at higher $[\mathrm{Fe} / \mathrm{H}]$ values relative to the change in slope in the solar vicinity. This is due to the high star formation rate and the short timescale for bulge formation. This fact was pointed out for the first time by MB90.

2. Where the IMF is flatter, the abundance trend of $\alpha$-elements vs. Fe is flatter as well, because SNeIa are fewer than in a Salpeter or steeper IMF. We also expect that in the Z00-4 case, which favours the creation of SNIa progenitors, Fe production will be enhanced.

3. For steeper IMFs (e.g. Scalo 1986; or Salpeter 1955), the nitrogen enrichment from low- and intermediate-mass stars is enhanced with respect to $\mathrm{O}$, which mostly comes from massive stars. As a result, the $[\mathrm{N} / \mathrm{O}]$ vs. $[\mathrm{O} / \mathrm{H}]$ plot should lie above the one of our fiducial model. Moreover, $\mathrm{N}$ primary production from low- and intermediate- mass stars should be enhanced as well, and the flattening of the above mentioned plot towards the latest evolutionary stages should be prolonged. In general, any model that favours the contribution of massive rather than low- and intermediate- mass stars (i.e. high $v$, low $\tau$ ) should predict a downturn of the $[\mathrm{N} / \mathrm{O}]$ vs. $[\mathrm{O} / \mathrm{H}]$ plot if compared to our fiducial model, and vice versa.

\subsection{Metallicity distribution of bulge giants}

Figure 3 shows the predicted MD for giant stars compared to the data from Z03 and FMR06a ${ }^{4}$.

The choice of a different IMF does not primarily influence the spread of the distribution but rather shifts its peak on the $[\mathrm{Fe} / \mathrm{H}]$ scale: in general, flatter IMFs result in distributions peaked at higher values of $[\mathrm{Fe} / \mathrm{H}]$. Since the star-forming phase is relatively short, massive stars will be important in the Fe enrichment; if we adopt the Scalo (1986) exponent (model Z00-4) rather than the Salpeter (1955) one (model Z00-3) for stars more massive than $2 M_{\odot}$, the resulting MD will change dramatically, moving the peak toward significantly lower $[\mathrm{Fe} / \mathrm{H}]$. Considerations on the MD allow us to exclude IMFs steeper than $x=0.95$, whereas plots for flatter IMFs are all consistent with the observed distributions. In fact, the MD obtained with the very flat $(x=0.33)$ IMF of case Z00-1 is almost undistinguishable from that calculated in case Z00-2, showing that the MD progressively becomes almost insensitive to the flattening of the IMF under a certain value of the index. This is maybe suggestive of a "saturation effect" in the Fe enrichment from Type II SNe. Namely, a flattening of the IMF below a certain value of the index $x$ does not produce a sensible increase of the number of massive stars, and moreover, the Fe mass ejected is assumed to be the same (i.e. does not increase as a function of mass) for all masses above $40 M_{\odot}$ (see Sect. 2).

If we choose a moderate star formation efficiency $(v=$ $2 \mathrm{Gyr}^{-1}$, comparable to that of the galactic halo), the predicted metallicity distribution is broadened and the number of both high- and low-metallicity stars is somewhat overestimated. The observed peak is poorly reproduced (both in height and position) for all of the MDs considered. In contrast, the extremely high value of $v=200 \mathrm{Gyr}^{-1}$ yields an extremely narrow distribution, with an excessive peak height (reaching 0.68 , but is truncated in the figure to preserve clarity).

${ }^{4}$ We must remark that we did not make an attempt to convolve the predictions with uncertainties. Since the observed distributions are probably broader than the "true" distribution, due to random errors, and may be offset due to systematic errors, their comparison is likely to be affected. 

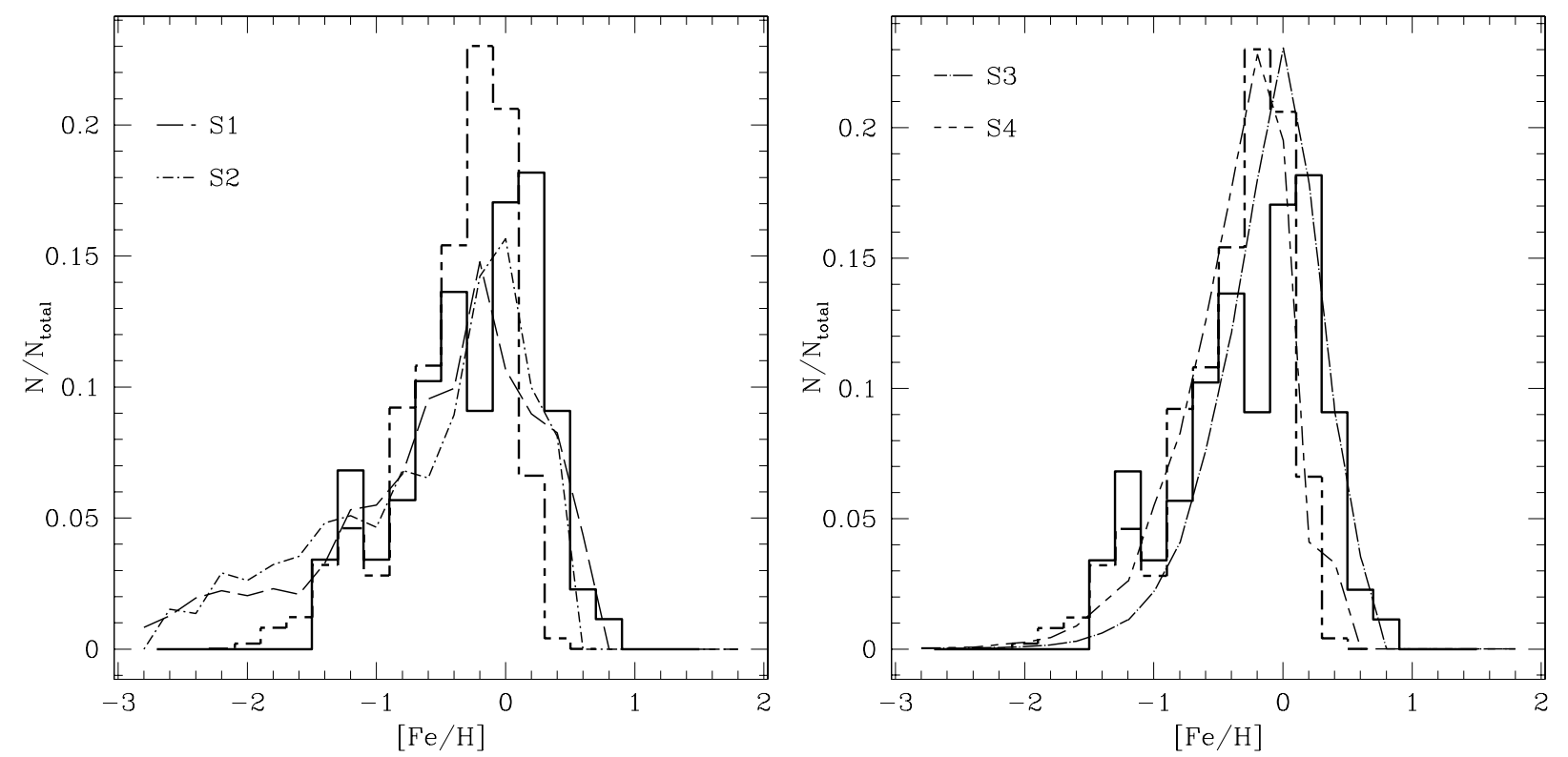

Fig. 4. Predicted MDs in models with $v=200 \mathrm{Gyr}^{-1}$ and $\tau=0.01 \mathrm{Gyr}$ (left panel) and in models with $v=2 \mathrm{Gyr}^{-1}$ and $\tau=0.7 \mathrm{Gyr}$, with the adoption of two different IMFs (see ext for details). Data are from Z03 (solid histogram) and FMR06a (dashed histogram).

A change in the infall timescale affects mainly the spread of the distribution and only slightly the position of the peak. The low-metallicity wing is especially sensitive to the value of $\tau$. The model with $\tau=0.01 \mathrm{Gyr}$ approaches a closed-box model, i.e. a model where all the gas is already present from the beginning (which would correspond to the limit $\tau=0$ ). The gas soon reaches high densities and is consumed very rapidly. Thus, the number of metal-poor stars is overestimated and the predicted distribution extends below the observed low-metallicity tail. This confirms the considerations of Z03, i.e. an equivalent (although less important) manifestation of the G-dwarf problem occurs also in the Galactic bulge. We also calculated the MD resulting from the adoption of a slightly longer infall timescale, namely $\tau=0.7 \mathrm{Gyr}$, that corresponds to the timescale $\tau_{\mathrm{H}}$ for collapse from halo to bulge in the model of Mollá et al. (2000). As the figure shows, the $\tau=0.7 \mathrm{Gyr}$ MD is at variance with observations, having a serious deficit of both metal-poor and metalrich stars and too high a peak with respect to both measured distributions (again higher than 0.5 but truncated in the figure).

The main conclusions which can be drawn for variations of a single parameter are:

1. Changing the IMF slope has the general effect of shifting the peak of the MD towards lower metallicities for steeper IMFs, and vice versa. For $x \leq 0.95$ such variations become much less evident. We want to point out that continuous wind such as that of the Samland et al. (1997) model cannot have the same effects of flattening the IMF, since outflows lower the effective yield, and thus if we had a continuous outflow we would need an even flatter IMF to reproduce the observed MD and abundance ratios. This was shown i.e. for the galactic disk by Tosi et al. (1999, their Fig. 7).

2. Lower star formation efficiencies give rise to broader MDs and viceversa. The same is true of shorter infall timescales, which additionally increase the number of low-metallicity stars.

3. The effect of both star formation efficiency and infall timescale on the position of the MD peak is negliglible.
We now explore whether any other combinations of parameters can fit the observed MD. Since the position of the peak is essentially only determined by the IMF, combining IMFs other than those of models Z00-1 and Z00-2 with different values of the other parameters would not be useful to reproduce the required MD. On the contrary, the effects of infall timescale and star formation efficiency could compensate each other; therefore, we also considered the following "supplementary" (S) models:

- Model S1: IMF like in model Z00-1, $v=200 \mathrm{Gyr}^{-1}$, $\tau=0.01$ Gyr.

- Model S2: same as in S1, but with IMF like in model Z00-2.

- Model S3: IMF like in model Z00-1, $v=2 \mathrm{Gyr}^{-1}$, $\tau=0.7 \mathrm{Gyr}$.

- Model S4: same as in S3, but with IMF like in model Z00-2.

In Fig. 4 are shown the resulting MDs for these supplementary models.

It is clear that while combining a high value of $v$ and a low value of $\tau$ exacerbates the bulge "G-dwarf problem", a longer formation timescale can combine with a milder star formation efficiency to give rise to a MD compatible with observations, and this is what happened in some of the previous models (Samland et al. 1997; Mollá et al. 2000). However, such a degeneracy cannot be pushed above a certain range of values. We investigated longer timescales and found out that it becomes progressively harder to mantain the required MD both in height and position, since the result of combining much longer timescales with efficiencies suitable to keep the required shape is to shift the distribution with the required shape toward lower metallicities, and vice versa, if we try to keep the predicted MD at the right position, its shape becomes too narrow, somewhat in contrast with the observed MDs. In any case, as we shall see, such a degeneracy is definitively broken when we take into account the evolution of abundance ratios (see next section).

For $\tau \geq 2$ Gyr, some star formation is predicted at detectable levels at the present time, contrarily to observations.

Therefore, we conclude that good agreement with the observed MD is achieved only if the bulge formed on a short 


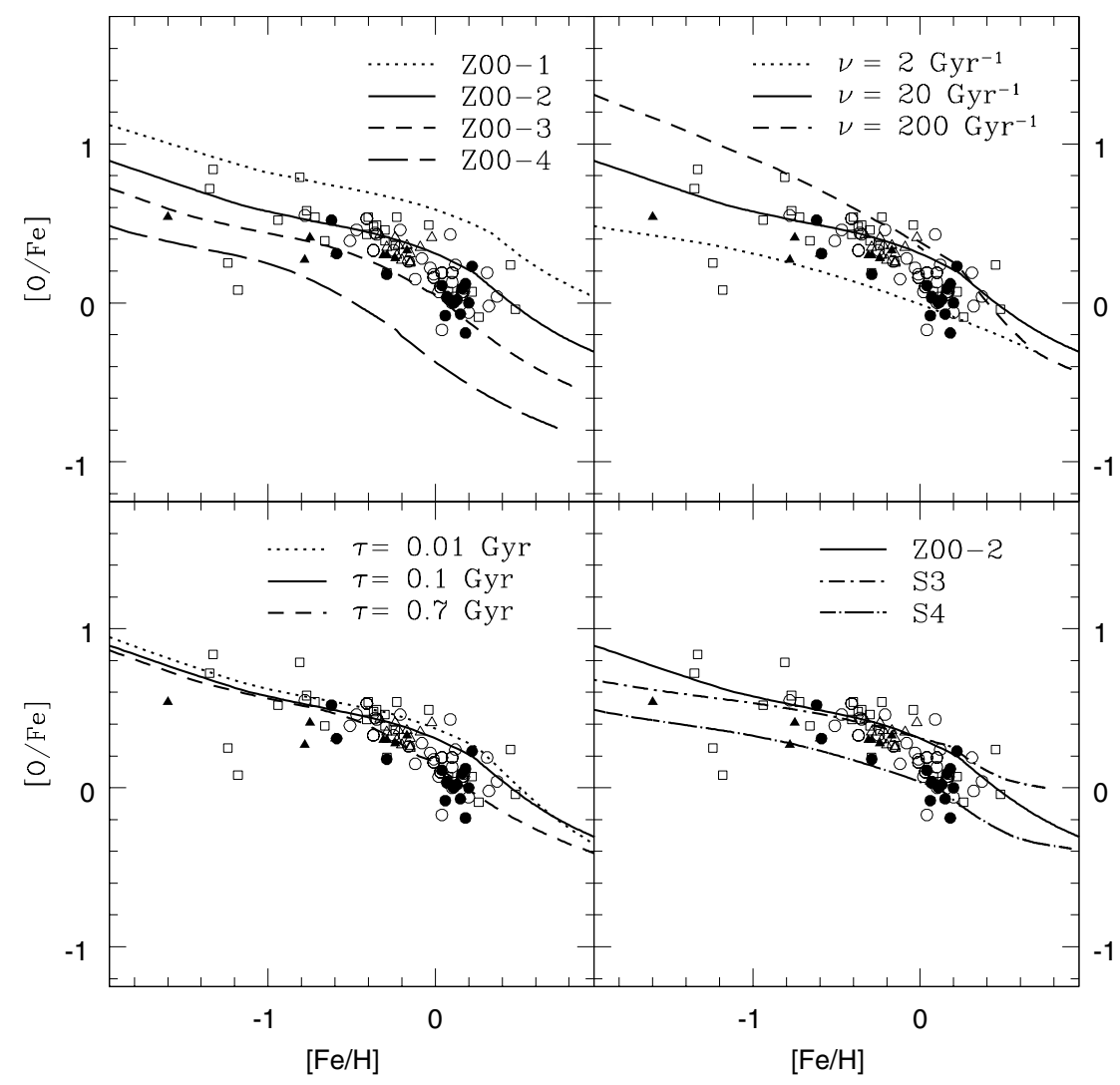

Fig. 5. Evolution of $[\mathrm{O} / \mathrm{Fe}]$ vs. $[\mathrm{Fe} / \mathrm{H}]$ in the bulge for different values of the IMF index (upper left panel; see text for details), star formation efficiency (upper right panel), and infall timescale (lower left panel). The lower right panel shows the results obtained combining a longer infall timescales and milder star formation efficiencies for two different IMFs (see text for details). Triangles represent data taken from the IR spectroscopic database (field stars are symbolized by filled triangles, stars in globular clusters by open triangles. See Sect. 3 for specific references). The open and filled circles represent the low- and high-quality data, respectively, from Zoccali et al. (2006). Finally, the squares are the data from Fulbright et al. (2006b) and Zoccali et al. (2006). timescale, with a flat IMF and with a rather high star formation efficiency.

\subsection{Evolution of abundance ratios}

\section{Oxygen}

Figure 5 shows the evolutionary plots of the $[\mathrm{O} / \mathrm{Fe}]$ abundance ratio with metallicity, which is the most sensitive to the adopted parameters. The models are compared to the data of the IR spectroscopic database, FMR06b and Z06. Each panel illustrates the effects of changing one of the model parameters as stated in Sect. 2. The last panel also shows the results obtained with models S3 and S4. We remind that when the bulk of SNeIa begin to explode, the $[\mathrm{O} / \mathrm{Fe}]$ ratio has a downturn and a knee in the $[\mathrm{O} / \mathrm{Fe}]$ vs. $[\mathrm{Fe} / \mathrm{H}]$ curve is expected.

The fiducial model provides a satisfactory fit to the existing measurements and predicts the correct amount of oxygen enhancement, even though the slope of the predicted plot in the fiducial model is slightly flatter than the observations for supersolar $[\mathrm{Fe} / \mathrm{H}]$. However, as we shall see, the fit to this abundance plot cannot be improved without violating other constraints. The amount of $\alpha$-enhancement crucially depends on the IMF index, and more in particular on the IMF of the Type II SN progenitors. The larger the number of massive stars, the higher the plateau at low metallicities. Model Z00-1 overproduces oxygen, and is not consistent with data. Figure 5 also allows us to exclude a Scalo (1986) exponent (model Z00-4) for massive stars in the bulge, since the corresponding evolution of $[\alpha / \mathrm{Fe}]$ vs. $[\mathrm{Fe} / \mathrm{H}]$ lies well below the observed data points. A Salpeter (1955) IMF (model Z00-3) cannot be excluded on the basis of these abundance ratios; however, it was ruled out by the MD plot.

Concerning the star formation efficiency, a value of $v=$ $20 \mathrm{Gyr}^{-1}$ is consistent with the observed abundance ratios, whereas lower or higher values seem to be at variance with the (few) lowest-metallicity data. The model with $v=200 \mathrm{Gyr}^{-1}$ predicts values of $[\mathrm{O} / \mathrm{Fe}]$ which are larger than those of the fiducial model at low metallicities, but then the gas is consumed very rapidly and star formation cease; this gives rise to a sharper decrease in the $[\mathrm{O} / \mathrm{Fe}]$ ratio at higher metallicities. The predicted slope is compatible with observations, however the absolute amount of oxygen enhancement is slightly overestimated, and in any case this model was already excluded on the basis of the MD (Fig. 3). It is noteworthy that even though the star formation stops much earlier in time for higher $v$ 's, the model trajectory still spans the same range in $[\mathrm{Fe} / \mathrm{H}]$ for all values of the star formation efficiency $v$. This is because at high values of the star formation efficiency, higher metallicities are attained in shorter times.

On the basis of the MD, we already saw that models with $v=2$ and $200 \mathrm{Gyr}^{-1}$ must be excluded; considerations derived using the $[\mathrm{O} / \mathrm{Fe}]$ abundance ratio provide a useful consistency check. We then suggest that a value of $20 \mathrm{Gyr}^{-1}$ can fit both the MD and the abundance ratios.

Instead, changing the infall timescale from 0.01 to $0.7 \mathrm{Gyr}$ has almost no effect on the evolution of the $[\mathrm{O} / \mathrm{Fe}]$ abundance ratio, and this holds also for other $\alpha$-elements, if we exclude a small improvement of the agreement with data in the $\tau=0.7 \mathrm{Gyr}$ case (which however, as well as the $v=200 \mathrm{Gyr}^{-1}$ case, is excluded by the MD plot). Therefore, there is no way of distinguishing between these models on the basis of considerations 
about abundance ratios, although we already excluded the cases of $\tau \ll 0.1 \mathrm{Gyr}$ and $\tau>0.1 \mathrm{Gyr}$ which yielded MDs in contrast with the observed ones.

Finally, we discuss the results obtained with the "supplementary" models S3 and S4, whose MD was consistent with observations. We can see that it is necessary to assume a very flat IMF (model S3) to avoid underestimating the $[\mathrm{O} / \mathrm{Fe}]$ ratio with the adoption of $\tau=0.7$ and $v=2 \mathrm{Gyr}^{-1}$, and the predicted slope is much flatter than the observed one. For even longer timescales, flattening the IMF is no longer sufficient to fit the data. This is due to the fact that Type Ia SNe have more time to pollute the ISM with Fe causing the plot to turn down, falling below the observations. Therefore, formation timescales longer than $\sim 1 \mathrm{Gyr}$ must be excluded in any case. This is the consequence of the time-delay model for Type Ia SNe in the case of different star formation histories, as already pointed out by MB90 and Matteucci (2000).

\section{Other $\alpha$-elements}

In Fig. 6 are shown the evolutionary plots of the abundance ratios of several $\alpha$-elements $(\mathrm{O}, \mathrm{Mg}, \mathrm{Si}$ and $\mathrm{Ca})$ to $\mathrm{Fe}$ versus $\mathrm{Fe}$ abundance, compared to the same datasets as $[\mathrm{O} / \mathrm{Fe}]$, with the exception of Z06. We also show a solar neighbourhood fiducial line for comparison, calculated according to François et al. (2004). A striking aspect of the predicted $[\alpha / \mathrm{Fe}]$ vs. $[\mathrm{Fe} / \mathrm{H}]$ relation is that the slope of the $[\alpha / \mathrm{Fe}]$ ratios changes only at $[\mathrm{Fe} / \mathrm{H}] \simeq 0$ for the fiducial model, in agreement with the data and at variance with the solar vicinity where this occurs at $[\mathrm{Fe} / \mathrm{H}]=-1.0$. Indeed, it is evident that a star formation history and an IMF such as those suitable for the solar neighbourhood give results which do not agree with the bulge observations, the general trend being to severely underestimate the data especially at $[\mathrm{Fe} / \mathrm{H}] \gtrsim-1$, when type I SNe, whose formation is favored by the steeper IMF, start polluting the ISM considerably.

It is worth noting that our fiducial model predicts that the slopes of $[\mathrm{O} / \mathrm{Fe}]$ and $[\mathrm{Mg} / \mathrm{Fe}]$ vs. $[\mathrm{Fe} / \mathrm{H}]$ are different, the latter being less steep. This is mainly due to the fact that whereas magnesium is mainly produced by a restricted range of stellar masses (around 20-25 $M_{\odot}$ ) oxygen is produced by a broad interval of stellar masses, (from 10 to $100 M_{\odot}$ ) the $\mathrm{O} / \mathrm{Fe}$ production ratio increasing with stellar mass. This different trend is present also in the solar vicinity data (see François et al. 2004) and is confirmed in the bulge by the observations of FMR06, which cover a larger range of metallicities than the IR spectroscopic database (i.e. $[\mathrm{Fe} / \mathrm{H}] \gtrsim 0$ and $[\mathrm{Fe} / \mathrm{H}] \lesssim-1$ ).

\section{Carbon and nitrogen}

Figures 7 and 8 show the evolutionary behaviours of $\mathrm{C}$ and $\mathrm{N}$, which, in contrast to the $\alpha$-elements are mainly produced by lowand intermediate-mass stars (and therefore on long timescales). The available dataset for carbon is limited to the measurements of the giant stars, which however are known to undergo mixing processes along the RGB, severly affecting the carbon abundances (in fact, we can immediately see that none of the models lie beneath the observations, since carbon is likely to be depleted during the evolution along the RGB). Hence, the comparison between models and observations cannot allow to firmly constrain the former and to reach strong conclusions in general. The observations can only provide a lower limit to any plausible model.

The behaviour of $[\mathrm{C} / \mathrm{Fe}]$ at low metallicities is largely affected by the assumed star formation efficiency, just as in the case of oxygen, whereas the curves do not show really discernible differences above the solar metallicity. A high value of the star formation efficiency $\left(v=200 \mathrm{Gyr}^{-1}\right)$ enhances the number of stellar generations and therefore accelerates the formation of intermediate-mass stars which are the main $\mathrm{C}$ producers, but again $[\mathrm{C} / \mathrm{Fe}]$ falls below the predictions of the fiducial model due to fast gas consumption, as was the case for oxygen. The steeply falling trend of $[\mathrm{C} / \mathrm{Fe}]$ until $\sim \operatorname{solar}[\mathrm{Fe} / \mathrm{H}]$ is due to the fact that the $\mathrm{C} / \mathrm{Fe}$ production factor is decreasing with decreasing mass for high-mass stars. In this case too, abundance data for metalpoor bulge stars can confirm or refute the conclusions reached on the basis of the MD, even though large values of $v$ seem again excluded.

A change in the IMF has a major impact on the contribution from low-mass stars. The plots show a bump at $[\mathrm{Fe} / \mathrm{H}]$ ranging from $\sim-0.4$ to 0.4 , which corresponds to the time at which the first intermediate mass stars contribute to $\mathrm{C}$ enrichment, with the exception of the Z00-1 IMF, for which the star formation ceases before the bulk of intermediate-mass stars have time to die. This bump is not visible in the solar neighbourhood, and its occurrence in the bulge is related to the strong metallicity-dependence of the adopted $\mathrm{C}$ yields for low- and intermediate-mass stars (Van den Hoek \& Groenewegen 1997), combined with the fact that in the bulge high metallicities are reached very early. The feature is more pronounced for larger values of $x$ because low- and intermediate-mass star formation is favoured by these steeper IMFs. This is particularly evident from the difference between the plots adopting a Salpeter (1955) and a Scalo (1986) IMF, respectively. Before the onset of the intermediate-mass dominated regime, the amount of $\mathrm{C}$ enrichment increases for flatter IMFs due to the enhanced $\mathrm{C}$ production from massive stars; on the contrary, when the massive star dominated regime ends, $\mathrm{C}$ production is increased remarkably for steeper IMFs due to the enhanced contribution from low- and intermediate-mass stars. Again, the Scalo IMF looks rather implausible, since the predicted trend almost lies beneath the data points, and this is likely to mean that $\mathrm{C}$ is underproduced in this model.

No appreciable change is seen when the adopted gas infall timescale is varied, with the exception of a slightly different position of the occurrence of the bump. The plots resulting from models S3 and S4 are also shown. Bearing in mind the paucity of data below $[\mathrm{Fe} / \mathrm{H}] \sim-0.5$, the model $\mathrm{S} 4$ predicts a trend which passes through the cluster of data points at $[\mathrm{Fe} / \mathrm{H}] \sim 0.0$, but since as we already stated $\mathrm{C}$ is likely to be affected by stellar evolution, this model probably underpredicts the $[\mathrm{C} / \mathrm{Fe}]$ ratio, again due to the Fe contribution from Type Ia SNe. Instead, model S3 leads to a larger $\mathrm{C}$ production and therefore keeps the consistency with the observations, the trend being only flatter than that of the fiducial model.

Nitrogen is the only element for which our reference model cannot yield a satisfactory agreement with data: in fact, it tends to lie under the observational points, following their lower envelope. This is true also for the solar vicinity. As we can see from Fig. 8, there is no way of reproducing the average trend of the $[\mathrm{N} / \mathrm{O}]$ abundance ratio with $[\mathrm{O} / \mathrm{H}]$ for single-parameter variations unless we adopt parameters which have proven to lead to results at variance with other constraints: e.g. a very low star formation efficiency, which results into an underproduction of oxygen with respect to nitrogen and was dismissed on the basis of the resulting MD (and also to some extent on the basis of the $[\mathrm{O} / \mathrm{Fe}]$ plot); or a steep IMF, favouring the formation of low- and intermediate-mass stars (which are supposed to produce the bulk 

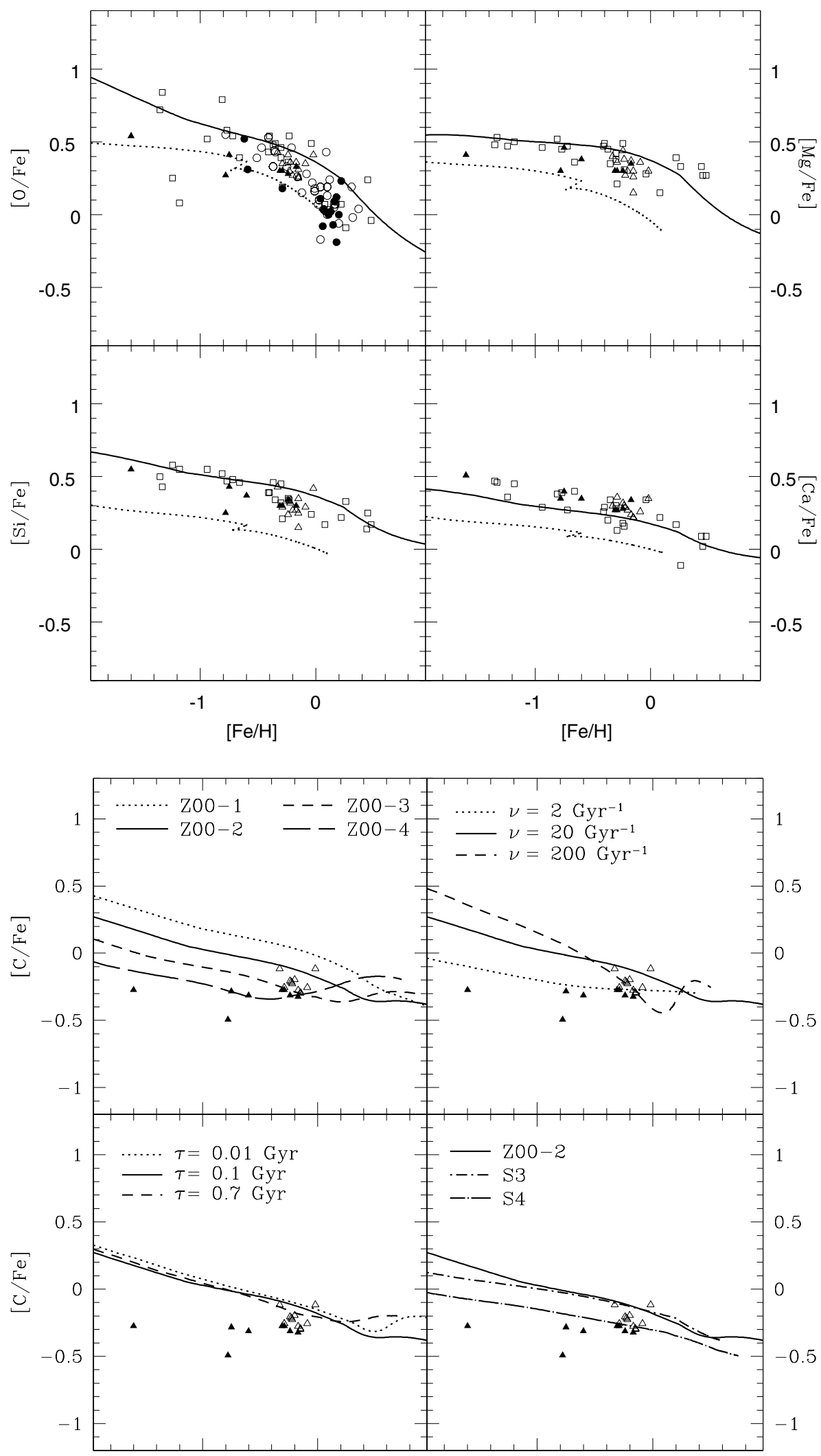

Fig. 6. Evolution of $[\alpha / \mathrm{Fe}]$ vs. $[\mathrm{Fe} / \mathrm{H}]$ in the bulge for $\mathrm{O}, \mathrm{Mg}, \mathrm{Si}, \mathrm{Ca}$ for the fiducial model (solid line). We also plotted a solar neighbourhood fiducial line (dotted line) for comparison. Data for oxygen are the same as in Fig. 5. For the other elements, data are taken from Fulbright et al. (2006b, open squares) and, for $\mathrm{Mg}$, from the IR spectroscopic database (field stars: filled triangles, clusters: open triangles. See text for detailed references).
Fig. 7. Evolution of $[\mathrm{C} / \mathrm{Fe}]$ vs. $[\mathrm{Fe} / \mathrm{H}]$ in the Galactic bulge for various choices of the IMF (upper left panel) star formation timescale (upper right panel), and infall timescale (lower left panel). The lower right panel shows the results obtained combining a longer infall timescale and a milder star formation efficiency for two different IMFs (see text for details). Data are from the IR spectroscopic database (field stars: filled triangles, clusters: open triangles. See text for detailed references). of $\mathrm{N}$ ), which did not reproduce either the MD or the evolution of $[\alpha / \mathrm{Fe}]$ vs. $[\mathrm{Fe} / \mathrm{H}]$.

A good agreement is instead achieved with the $\mathrm{S} 4$ case, since it achieves the same effect of a low efficiency of star formation (i.e. oxygen underproduction) while the longer formation timescale does not influence the evolution of abundance ratios. Models S3 still somewhat improves the match with data relative to the fiducial model, but the flatter IMF favours $\mathrm{O}$ enrichment with respect to model $\mathrm{S} 4$.

However, there is another way to obtain an acceptable fit to the observed abundances. In standard chemical evolution models nitrogen production from massive stars is supposed to be purely secondary, i.e. $\mathrm{N}$ is created starting from seed nuclei of $\mathrm{C}$ already present in the gas out of which the stars were born. 

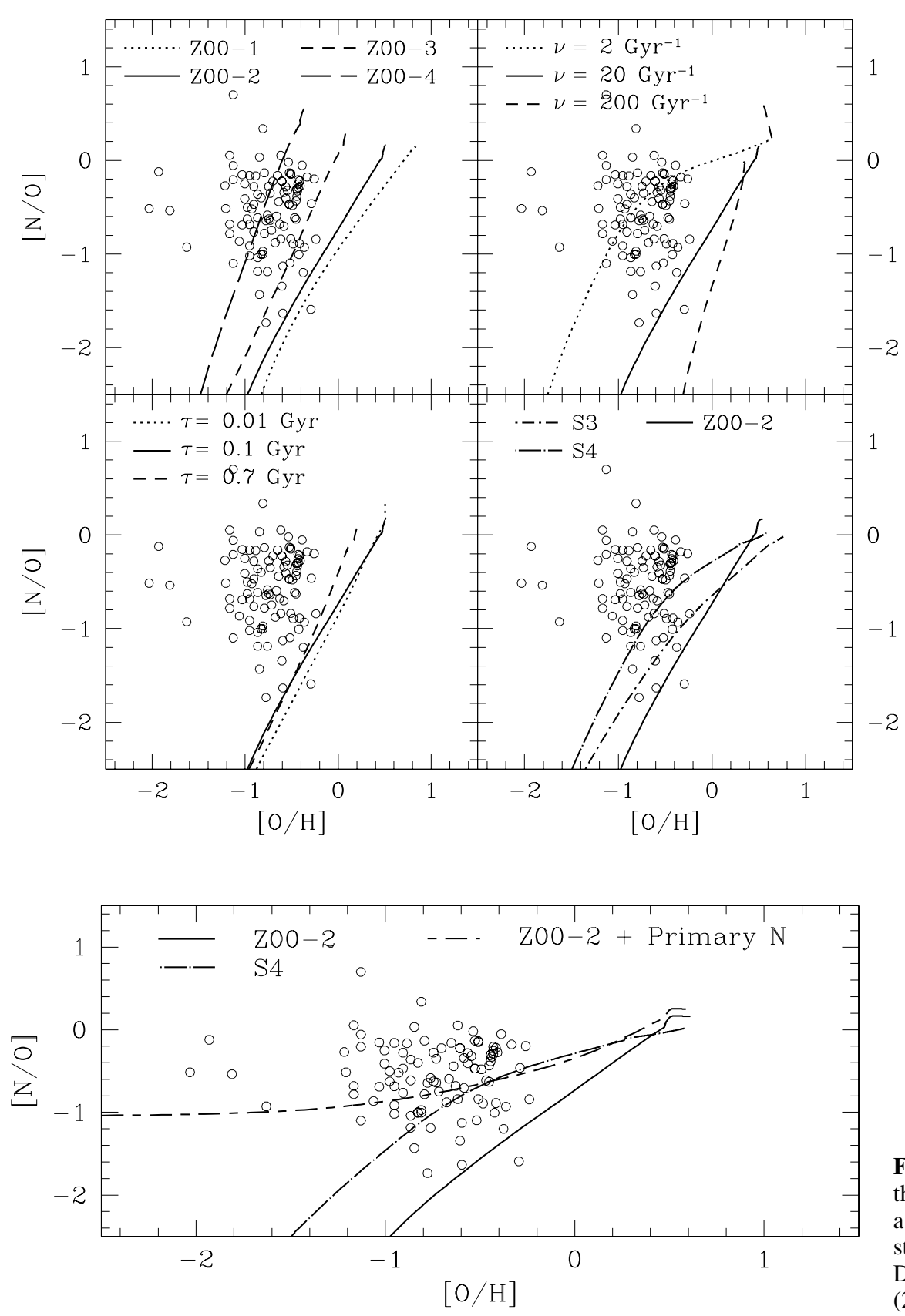

Fig. 8. Evolution of $[\mathrm{N} / \mathrm{O}]$ vs. $[\mathrm{O} / \mathrm{H}]$ in the Galactic bulge for various choices of the IMF (upper left panel) star formation timescale (upper right panel), and infall timescale (lower left panel). The lower right panel shows the results obtained combining a longer infall timescale and a milder star formation efficiency for two different IMFs (see text for details). The abundances measured from bulge $\mathrm{PNe}$ are taken from Górny et al. (2004).

Fig. 9. Evolution of $[\mathrm{N} / \mathrm{O}]$ vs. $[\mathrm{O} / \mathrm{H}]$ (right panels) in the Galactic bulge in our fiducial model compared to a model where primary production of $\mathrm{N}$ from massive stars is assumed (Matteucci 1986) and to model S4. Data for $\mathrm{N}$ and $\mathrm{O}$ in bulge $\mathrm{PNe}$ are from Górny et al. (2004).

Also low and intermediate mass stars are supposed to produce $\mathrm{N}$ in a secondary fashion but some primary $\mathrm{N}$ can be produced in intermediate mass stars during the third dredge-up episodes in conjunction with hot-bottom burning (Renzini \& Voli 1981; Van den Hoek \& Groenewegen 1997). The consequence of the secondary production is that the abundance of nitrogen should increase with metallicity in the earliest evolutionary phases, and that is what most evolutionary models of the Galaxy predict, not only for the bulge, but also for the solar neighbourhood (Ballero et al. 2006; Chiappini et al. 2005).

Therefore, we investigate what happens to the fiducial model if we assume that massive stars of all masses produce a constant amount $\left(0.065 M_{\odot}\right)$ of primary $\mathrm{N}$ at every metallicity. This hypothesis follows from the heuristic model of Matteucci (1986). The results are shown in Fig. 9 together with the plot of model $\mathrm{S} 4$. We see that this model seems to reproduce the average trend of the observations better than the standard model and, although it adopts an ad hoc assumption, it is useful to understand that some mechanism of primary production of nitrogen is likely to occur at any metallicity in massive stars. For example, Meynet \& Maeder (2002) first pointed out that stellar rotation can produce primary nitrogen in massive stars, and although Chiappini et al. (2003) demonstrated that their rotation yields are insufficient to produce the observed trend in the solar neighbourhood, this hypothesis is the most promising one, as shown by Chiappini et al. (2006), if we suppose an increase in rotational velocity at low metallicities (see Meynet et al. 2006; Hirschi et al. 2007). We thus suggest that a continuous primary $\mathrm{N}$ production from massive stars is necessary at any epoch, in analogy with what is required in the solar vicinity (see Chiappini et al. 2005, for an extensive discussion on this point). 
From Fig. 9 it can be also noticed that model $\mathrm{S} 4$ differs from model Z00-2 with primary $\mathrm{N}$ for $[\mathrm{O} / \mathrm{H}] \lesssim-1$, since while in the latter the $[\mathrm{N} / \mathrm{O}]$ ratio is almost independent of $[\mathrm{O} / \mathrm{H}]$, in the $\mathrm{S} 4$ model the plot curves down sharply at low metallicities due to secondary production from massive stars, and at $[\mathrm{O} / \mathrm{H}] \sim-1$ primary production from low- and intermediate-mass stars becomes dominant.

\section{Summary and conclusions}

In the present paper, we have computed chemical evolution models for the Galactic bulge where we have considered a scenario involving evolutionary timescales much faster than in the solar neighbourhood and in the halo, as the most probable one.

We have adopted new chemical yields suggested by François et al. (2004) which provided the best fit to the solar neighbourhood abundance trends.

New observations, unavailable at the time of earlier chemical evolution models (e.g., Matteucci \& Brocato 1990; Renzini 1993; Matteucci et al. 1999; Mollá et al. 2000) have been used to test these assumptions. The agreement between the new observations and our predictions is quite good, especially for the $\mathrm{Fe}$ abundance distribution and the $[\alpha / \mathrm{Fe}]$ trends.

Order-of magnitude changes in the main parameters determining chemical evolution (star formation efficiency, stellar initial mass function, timescale of gas collapse) were applied in order to explore the consequences of such changes on the predicted stellar metallicity distribution and trend of chemical abundances as a function of metallicity.

Our main conclusions can be summarized as follows:

- A short formation timescale combined with a high star formation efficiency fits both the observed metallicity distribution and chemical abundance ratios. This is typical of a star formation history in a burst regime, i.e. strongly concentrated in the first stages of the lifetime of the system and vanishing very quickly, in analogy with elliptical galaxies. We suggest an efficiency of star formation of the order of $20 \mathrm{Gyr}^{-1}$. However the assumption of a closed box must be given up since it gives rise to an excessive amount of low-metallicity stars (even though not as seriously as in the case of the Gdwarf problem for the solar neighbourhood). A finite, though small, accretion time is instead required. We suggest that this timescale should be of the order of $0.1 \mathrm{Gyr}$.

- There exists a sort of degeneracy between the gas infall timescale and the efficiency of star formation, in the sense that values of $\tau$ longer than $0.1 \mathrm{Gyr}$ can combine with values of $v$ lower than $20 \mathrm{Gyr}^{-1}$ in order to match the observed metallicity distribution. However, such a degeneracy is broken when we consider also the evolution of $[\mathrm{O} / \mathrm{Fe}]$ vs. $[\mathrm{Fe} / \mathrm{H}]$ and in any case values of $\tau$ longer than $1 \mathrm{Gyr}$ make it impossible to fit the observed metallicity distribution as well.

- An IMF flatter than those suitable for the solar neighbourhood properties, such as that of Scalo (1998) or Weidner \& Kroupa (2005), is necessary to reproduce the observational constraints. Namely, a value of $x=0.95$ for massive stars (even smaller than that proposed by Matteucci et al. 1999, which was $x=1.1$ ) gave the best overall fit. This can be theoretically understood if we note that the star formation in the Bulge proceeds like in a burst and there are several suggestions in the literature about a top-heavy IMF in starbursts (e.g. Baugh et al. 2005; Nagashima et al. 2005). Figer (2005) also finds a flat IMF in the Arches cluster near the Galactic center. The adopted flattening below $1 M_{\odot}$, as suggested by luminosity function measurements (Zoccali et al. 2000) does not affect significantly the abundance distribution. However, extremely flat IMFs (e.g. that of Z00-1 model), if extrapolated to massive stars, will lead to some degree of oxygen overproduction.

- The adoption of the fiducial model explains the behaviour of the different $[\alpha / \mathrm{Fe}]$ abundance ratios with metallicity very well, and predicts different slopes for different $\alpha$-elements, according to their nucleosynthesis. There is no need to invoke a second infall episode, as suggested by other authors (Mollá et al. 2000; Costa et al. 2005) to explain the observed values of the abundance ratios. We do not exclude however a second infall episode on a much longer timescale such as that hypothesized by Mollá et al. (2000) as it may help explain the presence of a very young stellar population confined to the very centre of our Galaxy, but it must involve a minor fraction of the bulge gas mass.

- A certain amount of primary nitrogen from massive stars might be required in order to reproduce the average trend of $[\mathrm{N} / \mathrm{O}]$ vs. $[\mathrm{O} / \mathrm{H}]$ with the fiducial model. The same conclusion was reached for the solar neighbourhood by Chiappini et al. (2005) and Ballero et al. (2006). The phenomenon of primary $\mathrm{N}$ production from massive stars as a result of rotation was studied by Maeder et al. (2005), Meynet et al. (2006) and Hirschi (2007) and this seems the most promising way (see Chiappini et al. 2006). These considerations followed from observations of $\mathrm{N}$ and $\mathrm{O}$ abundances in planetary nebulae; in order to firmly assess this point, abundance measurements in stars which have not experienced nitrogen self-enrichment are required, especially at low metallicities. In alternative, carbon measurements in the same planetary nebulae we have considered might allow an estimate of the amount of $\mathrm{N}$ synthesized by the PN progenitor.

- In the near feature new sets of empirical metallicity and abundance pattern distributions in different fields and based on high resolution spectroscopy will also become available. This will dramatically improve the chance of a more robust and quantitative comparison between theory and observations, with the ultimate goal of drawing the formation and chemical evolution history of the Galactic Bulge.

Acknowledgements. S. K. B. acknowledges A. Pipino for useful discussions and M. Zoccali for providing data in a timely manner.

\section{References}

Arimoto, N., \& Yoshii, Y. 1986, A\&A, 164, 260

Ballero, S. K., Matteucci, F., \& Chiappini, C. 2006, NewA, 11, 306 Baugh, C. M., Lacey, C. G., Frenk, C. S., et al. 2005, MNRAS, 356, 1191 Bertelli, G. P., Bressan, A. G., \& Chiosi, C. 1990, A\&A, 130, 279 Bertin, G., Saglia, R. P., \& Stiavelli, M. 1992, ApJ, 384, 423

Carretta, E., Cohen, J., Gratton, R. G., \& Behr, B. 2001, AJ, 122, 1469

Cavallo, R. M., Cook, K. H., Minniti, D., \& Vandehei, T. 2003, Proc. SPIE, 4834, 66, CCMV

Chiappini, C., Romano, D., \& Matteucci, F. 2003a, MNRAS, 339, 63

Chiappini, C., Matteucci, F., \& Meynet, G. 2003b, A\&A, 410, 257

Chiappini, C., Matteucci, F., \& Ballero, S. K. 2005, A\&A, 437, 429

Chiappini, C., Hirschi, R., Meynet, G., et al. 2006, A\&A, 449, L27

Cioffi, D. F., \& Shull, J. M. 1991, ApJ, 367, 96

Costa, R. D. D., Escudero, A. V., \& Maciel, W. J. 2005, to appear in the proceedings of the conference Planetary Nebulae as Astronomical Tools held in Gdansk, Poland [arXiv:astro-ph/0508693]

Cox, D. P. 1972, ApJ, 178, 143

Cuisinier, F., Maciel., W. J., Köppen, J., Acker, A., \& Stenholm, B. 2000, A\&A, 353,543 
Eggen, O. J., Lynden-Bell, D., \& Sandage, A. R. 1962, ApJ, 136, 748

Elmegreen, B. 1999, ApJ, 517, 103

Escudero, A. V., Costa, \& R. D. D. 2001, A\&A, 380, 300

Escudero, A. V., \& Costa, R. D. D., \& Maciel, W. J. 2004, A\&A, 414, 211

Ferreras, I., Wyse, R. F. G., \& Silk, J. 2003, MNRAS, 345, 1381

Figer, D. F. 2005, Nature, 434, 192

François, P., Matteucci, F., \& Cayrel, R., et al. 2004, A\&A, 421, 613

Fulbright, J. P., McWilliam, A., \& Rich, R. M.2005, NuPhA 758, 197

Fulbright, J. P., McWilliam, A., \& Rich, R. M. 2006a, ApJ, 636, 821, FMR06a

Fulbright, J. P., McWilliam, A., \& Rich, R. M. 2006b, ApJ, accepted [arXiv: astro-ph/0609087], FMR06b

Górny, S. K., Stasińska, G., Escudero, A. V., \& Costa, R. D. D. 2004, A\&A, 427, 231

Greggio, L., \& Renzini, A. 1983, A\&A, 118, 217

Grevesse, N.,\& Sauval, A. J.1998, SSRv 85, 161

Hirschi, R. 2007, A\&A, 461, 571

Hou, J., Chang, R., \& Fu C. 1998, Annals of Shanghai Observatory, Academia Sinica, 19, 96

Ibata, R. A., \& Gilmore, G. F. 1995, MNRAS, 275, 591

Iwamoto, K., Brachwitz, F., Nomoto, K., et al. 1999, ApJS, 125, 439

Jablonka, P., Martin, P., \& Arimoto, N. 1996, AL, 112, 1415

Kennicutt, R. C.Jr. 1998, ApJ, 498, 541

Kuijken, K., Rich, \& R. M. 2002, AJ, 124, 2054

Kodama, T. 1997, Ph. D. Thesis, University of Tokio

Liu, X. W., Luo, S. G., Barlow, M. J., Danziger, I. J., \& Storey, P. J. 2001, MNRAS 327, 141

Luna, G. J. M., \& Costa, R. D. D. 2005, A\&A, 435, 1087

Maeder, A., Meynet, G., \& Hirschi, R. 2005, in Cosmic Abundances as Records of Stellar Evolution and Nucleosynthesis, ed. T. G.Barnes III \& F. N.Bash, ASP Conf. Ser., 336, 79

Mannucci, F., Della Valle, M., Panagia, N., et al. 2005, A\&A, 433, 807

Maraston, C., Greggio, L., Renzini, A., et al. 2003, A\&A, 400, 823

Matteucci, F. 1986, MNRAS, 221, 911

Matteucci, F. 1992, ApJ, 397, 32

Matteucci, F. 1994, A\&A, 288, 57

Matteucci, F. 2000, in The Evolution of the Milky Way: Stars versus Clusters, ed F. Matteucci \& F. Giovannelli, ASSL 255 (Kluwer Academic Publishers), 3

Matteucci, F., \& Greggio, L. 1986, A\&A, 154, 279

Matteucci, F., \& Tornambé, A. 1987, A\&A, 185, 51

Matteucci, F., \& Brocato, E. 1990, ApJ, 365, 539, MB90

Matteucci, F., \& Recchi, S. 2001, ApJ, 558, 351

Matteucci, F., Romano, D., \& Molaro, P. 1999, A\&A, 341, 458, MRM99

McWilliam, A., \& Rich, R. M. 1994, ApJS, 91, 749, MR94

McWilliam, A., \& Rich, R. M. 2004, in Origin and Evolution of the Elements, from the Carnegie Observatories Centennial Symposia, Carnegie Observatories Astrophysics Series, ed. A. McWilliam \& M. Rauch, 38
McWilliam, A., Rich, R. M., \& Smecker-Hane, T. A. 2003, ApJ, 592, L21 Melèndez, J., Barbuy, B., Bica, E., et al. 2003, A\&A, 411, 417 Meynet, G., \& Maeder, A. 2002, A\&A, 390, 561

Meynet, G., Ekström, S., \& Maeder, A. 2006, A\&A, 447, 623

Mollá, M., Ferrini, F., \& Gozzi, G. 2000, MNRAS, 316, 345

Nagashima, M., Lacey, C. G., Baugh, C. M., Frenk, C. S., \& Cole, S., MNRAS, 358,1247

Nomoto, K., Thielemann, F. K., \& Yokoi, K. 1984, ApJ, 286, 644

Nussbaumer, H., Schmid, H. M., Vogel, M., \& Schild, H. 1988, A\&A, 198, 179

Origlia, L., \& Rich, R. M. 2004, AJ, 127, 3422

Origlia, L., Rich, R. M., \& Castro, S. 2002 AJ, 123, 1559

Origlia, L., Ferraro, F. R., Bellazzini, M. \& Pancino, E. 2003, ApJ, 591, 916

Origlia, L., Valenti, E., \& Rich, R. M. 2005, MNRAS, 256, 127

Ortolani, S., Renzini, A., Gilmozzi, R., et al. 1995, Nature, 377, 701

Peimbert, M., \& Serrano, A. 1980, RMxAA, 5, 9

Pompéia, L., Barbuy, B., \& Grenon, M. 2003, ApJ, 592, 1173

Ramírez, S. V., Stephens, A. W., Frogel, J. A., \& DePoy, D. L. 2000, AJ, 120, 833

Renzini, A. 1993, in Galactic Bulges, ed. H. DeJonghe \& H. J.Habing (Dordrecht, Kluwer Academic Publishers), IAU Symp., 153, 151

Renzini, A. 2002, in Chemical Enrichment of Intracluster and Intergalactic Medium, ed. R. Fusco-Femiano \& F. Matteucci, ASP Conf. Ser., 253, 331 Renzini, A., \& Voli, M. 1981, A\&A, 94, 175

Rich, R. M. 1988, AJ, 95, 828

Rich, R. M., \& Origlia, L. 2005, ApJ, 634, 1293

Sadler, E. M., Rich, R. M., \& Terndrup, D. M. 1996, AJ, 112, 171

Salpeter, E. E. 1955, ApJ, 121, 161

Samland, M., Hensler, G., \& Theis, C. 1997, ApJ, 476, 544

Sarajedini, A., \& Jablonka, P. 2005, AJ, 130, 1627

Scalo, J. M. 1986, FCPh 11, 1

Searle, L., \& Zinn, R. 1978, ApJ, 225, 357

Stasińska, G., Tylenda, R., Acker, A., \& Stenholm, B. 1991, A\&A, 247, 173

Terndrup, D. M.1988, AJ, 96, 884

Tosi, M., Steigman, G., Matteucci, F., \& Chiappini, C. 1998, ApJ, 498, 226

Van den Hoek, L. B., \& Groenewegen, M. A. T. 1997, A\&AS, 123, 305

Walton, N. A., Barlow, M. J., Clegg, R. E. S. 1993, in Galactic Bulges, eds. H. DeJonghe, \& H. J.Habing (Dordrecht Kluwer Academic Publishers), IAU Symp. 153, 337

Webster, B. L.1984, ASAu Proc., 5, 535

Weidner, C., \& Kroupa, P. 2005, ApJ, 625, 754

Whitford, A. E. 1978, ApJ, 226, 777

Woosley, S. E., \& Weaver, T.A, 1995, ApJS, 101, 181

Wyse, R. F. G. 1998, MNRAS, 293, 429

Zoccali, M., Cassisi, S., Frogel, J. A., et al. 2000, ApJ, 530, 418, Z00

Zoccali, M., Barbuy, B., Hill, V., et al. 2004, A\&A, 423, 507

Zoccali, M., Lecureur, A., Barbuy, B., et al. 2006, A\&A, 457, L1

Zoccali, M., Renzini, A., Ortolani, S., et al. 2003, A\&A, 399, 931, Z03 